\title{
PROCEEDINGS OF THE PATHOLOGICAL SOCIETY OF GREAT BRITAIN AND IRELAND
}

The 143rd meeting of the Society was held at the Ninewells Hospital and Medical School, University of Dundee on 8,9 and $10 \mathrm{July} 1981$

\section{SYNOPSES OF PAPERS OF MICROBIOLOGICAL INTEREST}

These synopses should not be cited as references in published work without the permission of the authors.

\section{A QUantitative analysis of leUCOCYTE Chemotaxis}

R. A. Brown, D. M. Parham, A. J. Robertson, R. C. Potts and J. Swanson Beck

Department of Pathology, Ninewells Hospital and Medical School, Dundee DDI 9SY

In this paper we describe a method for measurement of leucocyte chemotaxis in a Boyden chamber with a Quantimet 720 image-analysing computer to determine the spatial distribution of the cells within the filter. The data so obtained were then analysed on the assumption that cell movement may be described by a 3-dimensional random walk with drift in the direction of the chemo-attractant concentration gradient. This permits the estimation of two fundamental constants and their standard errors.

\section{THE TOXIC EFFECTS OF CYCLOSPORIN A IN NORMAL RATS}

\author{
A. W. Thomson, P. H. Whiting, J. T. Blair, R. J. L. Davidson \\ and J. G. Simpson \\ Departments of Pathology and Chemical Pathology, University of Aberdeen, \\ Foresterhill, Aberdeen AB9 $2 Z D$
}

The fungal metabolite cyclosporin A (CyA) is an important new pharmacological immune suppressant affecting humoral and cell-mediated immunity by a selective inhibitory action on $\mathrm{T}$ cells. Clinical trials of the drug have been hampered by reports of its toxic effects. We have studied the potential toxicity of orally-administered CyA in normal rats, where drug dosage of $50 \mathrm{mg} / \mathrm{kg} / 48 \mathrm{~h}$ is immunosuppressive in terms of the humoral response to sheep red blood cells and graft-versus-host reactivity. Increasing the administration of CyA to $100 \mathrm{mg} / \mathrm{kg} / 24 \mathrm{~h}$ causes a variety of pathological effects. The animals show hair loss and a failure to gain weight. There is a progressive rise in serum urea, serum creatinine and urinary $\mathrm{N}$-acetyl- $\beta$-D-glucosaminidase with a fall in urea clearance and structural evidence of proximal tubular cell damage. Hypoalbuminaemia and hyperbilirubinaemia are accompanied by a fall in serum aspartate aminotransferase, changes in serum alkaline phosphatase and fatty change in hepatocytes. Haematological effects include reduced marrow cellularity and lymphopenia with the appearance of abnormal lymphocytes in the peripheral blood. These changes, which are at least partially reversible, shed further light on the toxic effects observed when CyA is administered in clinical transplantation. 


\section{IN-VITRO EFFECTS OF TETRACYCLINE DRUGS ON MITOGEN-STIMULATED LYMPHOCYTE GROWTH}

R. C. Potts, A. MacConnachie, A. A. Hassan, A. J. Robertson, L. Jones, J. H. Gibbs, R. A. Brown and J. Swanson Beck Department of Pathology, Ninewells Hospital and Medical School, Dundee DD1 9SY

Recent reports have suggested that some tetracycline drugs can suppress PHA and PWM-induced lymphocyte growth. The present study has shown that these drugs do not interfere with the initiation of the blastogenic response but, rather, reduce the replicative growth in established cultures. There was considerable individual variation in dose-response curves in studies on cells of a group of healthy volunteers.

\section{CONTRIBUtion BY THE SPLEEN AND LUNGS TO THE KINETICS OF BLOOD CLEARANCE OF COLLOIDS}

R. G. White

Department of Pathology, University of Cambridge, Cambridge

The kinetics and effectiveness of the blood clearance of antigen-antibody complexes is crucial for the onset and course of diseases due to the peripheral deposition of immune complexes. The traditional post-Aschoff view is that clearance of blood-borne colloids depends on the phagocytic function of the "reticulo-endothelial system". Indeed, the kinetics of clearance in defined conditions of colloid dosage have been regarded as a valid measure of phagocytic function. The role of endocytosis by macrophages in blood-colloid clearance is challenged on two grounds: (1) the kinetics are influenced by nonmacrophage segregation of colloid into the lungs and (2) the conditions for segregation of blood colloids into the spleen are different from those on the liver. The application of these concepts to a study of the kinetics of blood clearance of colloids by the domestic fowl was discussed in relation to the concept that splenic segregation is by a process of membrane capping by vascular endothelium and dendritic reticular cells and that pulmonary segregation depends on surface trapping by thrombocytes.

\section{What do immune COMplex assays measure?}

$$
\text { P. J. Gallagher, N. J. Goulding and D. B. Jones }
$$

\section{Department of Pathology, Southampton University General Hospital, Southampton SO9 $4 X Y$}

More than 50 techniques have been described for measuring circulating immune complexes but no single method has gained universal acceptance. Different assays applied to the same serum sample can produce widely divergent results and standardisation is a recurrent problem. Using alkaline and heat-aggregated preparations of Cohn fraction II human IgG as standards, we compared two of the more commonly used methods, the $\mathrm{Clq}$ binding technique and the Raji cell immunoassay.

The aggregates were fractionated on a Sephadex AcA $22(1.5 \times 81-\mathrm{cm})$ column and markers for $7 \mathrm{~S}$ and $19 \mathrm{~S}$ run concurrently. Both aggregates produced a peak in the $19 \mathrm{~S}$ region but the alkaline-aggregated preparation contained substantially more material in the fractions between $7 \mathrm{~S}$ and 19S. Conversely, more monomeric immunoglobulin was present in the heat aggregates. Both assays were efficient at detecting material in the $19 \mathrm{~S}$ region but $\mathrm{Clq}$ binding was superior for the intermediate fractions. Neither test gave a positive result with monomeric (7S) immunoglobulin.

We concluded that alkaline aggregates of immunoglobulin produced a wider range of 
particle size than heat-treated preparations and that the Clq binding test is more efficient than the Raji immunoassay in detecting complexes between $7 \mathrm{~S}$ and $19 \mathrm{~S}$ in size.

\section{Allergic aspergillosis of the para-nasal sinuses}

D. Lamb, J. Miller and A. Johnston

\section{Departments of Pathology, Respiratory Unit and Department of ENT Surgery,} University of Edinburgh, Edinburgh EH8 $9 A G$

There are numerous reports in the literature of involvement of para-nasal sinuses by Aspergillus fumigatus and occasionally other Aspergillus species. These reports describe two broad groups, aspergilloma non-invasive, but occasionally expanding mycelial masses, and invasive aspergillosis, as an opportunistic infection associated with immune suppression.

We have recently identified a group of five cases of chronic sinusitis which on pathological examination of the contents of the sinus showed appearances similar to that seen in the mucus plugs of allergic broncho-pulmonary aspergillosis of the lung. The inspissated debris included mucus, proteins and cellular debris, eosinophils, Charcot-Leyden crystals and variable, usually small, amounts of hyphal material. These patients have been studied with regard to aspergillus skin and precipitin tests but in only one case was Aspergillus fumigatus cultured from the sinus. This case also had allergic broncho-pulmonary aspergillosis.

In the past, fungal involvement of the sinuses has not been clearly classified. We think it appropriate that involvement of the upper respiratory tract by Aspergillus species be classified in a similar manner to that of pulmonary involvement. We believe that the cases presented represent the first description of allergic aspergillosis of the para-nasal sinuses.

\section{SAPROPHYTIC INVASION OF INFARCTED PULMONARY TISSUE BY ASPERGILLUS SPECIES}

D. R. Buchanan and D. Lamb

\section{Department of Pathology, University of Edinburgh Medical School, Edinburgh EH8 $9 A G$}

Involvement of pulmonary tissue by Aspergillus species is usually considered to include three main patterns: development of a fungal ball or mycetoma in a pre-formed cavity, allergic broncho-pulmonary aspergillosis and true invasive aspergillosis as an opportunistic infection in patients with lowered tissue defences.

We present five cases of a form of pulmonary involvement of Aspergillus species which has previously received only passing reference in the literature. All five are examples of saprophytic invasion of infarcted pulmonary tissue. The characteristic features are widespread invasion of the dead tissue of fungal hyphae with, in three cases, liquefaction of the dead tissue with production of cavitating lesions.

Two cases were from incidental autopsy findings, two cases presented as isolated peripheral pulmonary opacities and were resected as possible tumours and one case presented as a complication of a pneumonic episode and raised the clinical possibility of primary aspergillus pneumonia. Only this latter case had mycological confirmation of the presence of Aspergillus fumigatus. It may be that examples of such saprophytic invasion of extensive pulmonary infarcts are the basis for some reported cases of primary aspergillus pneumonia. 


\title{
23. DifFERENTIAL TYPING OF STRAINS OF SHIGELLA SONNEI IN DUNDEE (1971-6)
}

\author{
D. C. Old and S. Helgason
}

\section{Department of Bacteriology, Ninewells Hospital and Medical School, Dundee DDI 9SY}

An epidemiological study of Sonne dysentery in Dundee during the years 1971-1976 was based on the examination of 1420 isolates of Shigella sonnei by colicine typing, antibiogram testing, biotyping and resistotyping. Analysis of the data for isolates recovered from almost 300 distinct episodes indicated that some characters associated with colicine type or antibiogram profile showed a high degree of variability in vivo and in vitro. Though biotype markers were stable, biotyping was poorly discriminatary. The technique affording greatest discrimination of types and least variability of markers in vivo and in vitro was resistotyping.

A multiple-typing approach was applied in which information from resistotyping, used as the primary differential typing system, was supplemented with data from colicine typing, antibiogram testing and biotyping, used as ancillary typing systems. At least 19 different "multiple types" of $S$. sonnei were recognised with the combined use of data from the four typing systems.

The multiple-typing approach was used to advantage in the analysis of: (i) isolates of different types from person(s) in a single episode; (ii) an outbreak involving primarily day nurseries and extending over a 10-month period, in which isolates of three distinct multiple types of $S$. sonnei were simultaneously present.

Multiple typing afforded some insight into the emergence, persistence, disappearance and co-existence of different types of $S$. sonnei in the community during the 6 years of study.

\section{DIFFERENTIATION OF STRAINS OF ESCHERICHIA COLI: MULTIPLE-TYPING APPROACH}

Pamela B. Crichton and D. C. Old

\section{Department of Bacteriology, Ninewells Hospital and Medical School, Dundee DD19SY}

Because of the vast number of antisera required, complete serological analysis of strains of Escherichia coli is impractible for all but national reference laboratories; there is an obvious need for other methods of characterising $E$. coli strains.

Results of tests with 21 substrates, examined for their ability to discriminate 671 cultures of $E$. coli collected from different sources, indicated that many of them should be discarded, because either their interpretation was unacceptably difficult or they provided little discrimination. Biotype profiles of strains were obtained from reactions in four "primary" and six "secondary" tests. Observations on 1444 cultures showed that biotype characters were stable in vivo and after storage on non-selective medium for many years.

A very fine degree of strain discrimination among 835 clinical isolates of $E$. coli was provided by biotyping ( 10 characters) and resistotyping ( 8 characters); used in combination, these systems identified relationships among sequential cultures from 182 of 200 patients, even in the absence of serotyping or, on occasion, when strains had undergone in-vivo variation in one or more typing characters. Although of limited discriminating value when used in isolation, patterns of mannose-sensitive and mannose-resistant haemagglutinating activity, antibiotic resistance, and colicin production and sensitivity aided strain identification when used along with biotyping and resistotyping. The use of a combination of these typing techniques should guarantee accurate characterisation of $E$. coli strains in epidemiological studies and should be within the resources of most laboratories. 


\section{RELATIONSHIPS AMONG PHAGE-TYPED STRAINS OF SALMONELLA TYPHIMURIUM REVEALED BY BIOTYPING}

Ruth M. Barker and D. C. Old

\section{Department of Bacteriology, Ninewells Hospital and Medical School, Dundee DDI 9SY}

Biotyping of more than $\mathbf{4 0 0 0}$ cultures of phage-typed strains of Salmonella typhimurium has shown that it is an excellent ancillary method for differentiating strains of that serotype. Studies of three groups of strains illustrated the point that epidemiological relationships of cultures from separate outbreaks may often be confused unless they are typed by more than one method.

Biotyping of 551 cultures of $S$. typhimurium of phage type 141 showed that three distinct clones, of biotypes $1 \mathrm{f}, 9 \mathrm{f}$ and $31 \mathrm{bd}$, were present among strains isolated in Scotland from 1965 to 1977.

Chloramphenicol-resistant strains of $S$. typhimurium of phage types 49, 204 and 193 were known to be related, from an analysis of the plasmids they carried. Biotyping revealed a similar relationship between wider resistance-type groups of strains of these phage types that were primary biotype 26 and suggested possible lines of descent for other strains of phage type 193.

S. typhimurium strains of a rare phage type (RDNC 2) were the cause of two milk-borne outbreaks in the Tayside region. The first, involving 32 patients, occurred from April 1975 to June 1976 and the second, with 20 cases, occurred 16 months later. On each occasion, the type of $S$. typhimurium that affected the human cases was isolated also from bovine sources. In addition to the similarity in phage type, strains from both episodes were of the same biotype, $17 \mathrm{a}$, also a relatively uncommon one. Investigation of the biotype of other strains causing infection in the area immediately before the first outbreak suggested that the RDNC 2 line derived from a strain of phage type 6. Cultures of both phage types were also similar in their resistance to antibiotics.

\section{OBSERVATIONS ON THE MORPHOLOGICAL EFFECT OF PENICILLIN ON GONOCOCCI GROWING ON SOLID MEDIUM}

\section{J. J. Jamil, S. Hafiz and M. G. McEntegart}

Department of Medical Microbiology, University of Sheffield Medical School, Sheffield S10 2RX

In the course of studies on the MIC and MBC of penicillin for gonococci on solid and in liquid media we took the opportunity to examine colonies by scanning electronmicroscopy. The results were unexpected and have interesting implications for the penetration of penicillin into the colony and for the homogenicity of the organisms within the colony as judged by their penicillin sensitivity.

\section{GROUP-B STREPTICOCCI-GASTROINTESTINAL ORGANISMS?}

C. S. F. Easmon, A. Tanna, P. Munday* and S. Dawson $\dagger$

Departmens of Bacteriology and Genito-urinary Medicine, $\dagger$ St Mary's Hospital and Medical School, London W2 1PG and

Division of Communicable Diseases, ${ }^{*}$ Clinical Research Centre, Northwick Park Hospital, Watford Road, Harrow, Middlesex HA1 $3 U \mathrm{UJ}$

Matched perianal swabs, rectal swabs and faecal samples from a group of male homosexual patients attending a clinic for sexually transmitted disease were examined for the presence of group-B streptococci (GBS). GBS isolation rates were as follows: perianal skin 31/115 (27\%), 
rectal mucosa $18 / 72(25 \%)$ and faeces $7 / 115(6 \%)$. The isolation of GBS from faeces was similar to that obtained from faecal samples sent to the laboratory for routine investigation $(5 \%)$. Although there was no difference in GBS isolation rates from rectal and perianal swabs, the latter did show heavier colonisation. These results suggest that gastrointestinal GBS carriage is in the main limited to the rectum and anal canal and that this may represent contamination from perianal skin.

\section{PROTECTIVE EFFECT OF CAERULOPLASMIN IN ENDOTOXIC SHOCK}

\section{J. M. Stark, H. C. Ryley and Barbara M. Darke}

Department of Medical Microbiology, Welsh National School of Medicine, Heath Park, Cardiff CF4 $4 X N$

Preparations of the human plasma-protein caeruloplasmin, had a protective effect against endotoxin in endotoxin in endotoxin-sensitive mice. The mice sensitised by BCG given intravenously 14 days beforehand, were exquisitely sensitive to endotoxin. Caeruloplasmin $(1-2 \mathrm{mg})$ given as a single dose intraperitoneally immediately after an intravenous dose of endotoxin $(2-10 \mu \mathrm{g})$ delayed death by several hours and in some instances allowed survival after an otherwise invariably fatal dose of endotoxin.

The protein was given on the assumption that its antioxidant properties would protect the host against the toxic oxygen derivatives (free oxygen radicals and singlet oxygen) released from phagocytes increased in number after BCG and further stimulated by endotoxin.

These findings have implications in the development of endotoxic shock, in the Shwartzmann reaction and in the use of endotoxin as an immunological adjuvant.

\section{TRIMETHOPRIM R-PLASMID RESISTANCE IN EDINBURGH}

\section{S. G. B. AMYES \\ Department of Bacteriology, University of Edinburgh Medical School, Edinburgh EH8 $9 A G$}

Three years after the introduction of trimethoprim in Britain, urinary pathogens were isolated that possessed R-plasmid-mediated resistance to this drug. R-plasmid-determined trimethoprim resistance was initially uncommon but the proportion of urinary bacteria possessing these plasmids has been steadily increasing in Britain in recent years.

In some Edinburgh hospitals the incidence of trimethoprim resistance among urinary isolates is high and the contribution made by trimethoprim $\mathbf{R}$ plasmids is the highest reported anywhere. The plasmids responsible are not all identical; this indicates that there is dissemination of the trimethoprim-resistance gene through the clinical population and the high proportion is not simply due to cross infection. In a controlled investigation with patients who provided sensitive isolates, it was found that a major contributing factor for the selection of the trimethoprim $\mathbf{R}$ plasmids is related to the use of ampicillin rather than trimethoprim. This is further supported by the high degree of linkage between trimethoprim and ampicillin on the $R$ plasmids. Once established, the plasmids could persist for many months in the absence of selective therapy. The eradication of bacteria possessing $\mathbf{R}$ plasmids conferring trimethoprim and ampicillin resistance was achieved only by the administration of unrelated antibacterial chemotherapy. More recently, there has been a tendency for trimethoprim R-plasmid resistance to lose its autotransferability and this may indicate its transposition into the chromosome. 
30. EFFECT OF AMPICILLIN AND CEFACLOR ON ACTIVITY OF BLOOD AND GINGIVAL CREVICULAR NEUTROPHIL MYELOPEROXIDASE

\author{
M. J. Kowolik, M. Grant and J. A. Raeburn \\ Departments of Oral Medicine and Human Genetics, University of Edinburgh, \\ Edinburgh
}

Myeloperoxidase, MPO (E.C.1.11.1.7), is important for the bactericidal function of neutrophils via hydrogen-peroxide-mediated oxidations in the presence of a halide. Blood neutrophil MPO activity is sensitive to antimicrobial drugs in vivo and in vitro. The aim of this study was to determine the sensitivity of blood and gingival crevicular neutrophil MPO to ampicillin and cefaclor in vivo.

A single 500-mg oral dose of either drug was given to healthy volunteers. Samples of venous blood and gingival crevicular cells were obtained immediately before drug ingestion, at $30-\mathrm{m}$ intervals for $2 \mathrm{~h}$ and thereafter hourly until $4 \mathrm{~h}$. Neutrophil MPO activity was assayed by the method of Klebanoff. Serum drug levels were also determined at each time interval.

In both cell populations, ampicillin depressed MPO activity while cefaclor enhanced it, the effect being significantly more marked in the gingival neutrophils. A sequential latency between the peak serum drug level, the blood neutrophil MPO response and the crevice neutrophil MPO response was seen. The clinical significance of these effects for patients on short or long-term antimicrobial therapy is not known, but the increased sensitivity of the gingival cell MPO provides evidence for a hypothesis of neutrophil subpopulations in the gingival crevice.

\title{
31. ThE ANTIBODY RESPONSE OF RABBITS TO CANDIDA ALBICANS
}

D. Parratt and P. F. H. Dawes

Department of Bacteriology, Ninewells Hospital and Medical School, Dundee DD1 9SY

Rabbits have been colonised with Candida albicans by feeding live suspensions of organisms to them, during intermittent chloramphenicol therapy. The aim has been to reproduce a type of infection similar to that which occurs in man, and which can be regarded as a low-grade colonisation, so that the immune response to the organism can be studied and manipulated. As a first experiment, the seum antibody response of suitably colonised animals was studied. Three of five animals showed stable colonisation during a 2-month period and their anticandida antibody level was remarkably constant. In another animal, after an initial period of colonisation, the organism was eliminated and in this case the antibody levels were closely correlated with the presence or absence of the organism throughout the experiment. Control animals showed no significant antibody response to $C$. albicans, except that one animal at the end of the experiment became naturally colonised, and produced antibody. The effect of two separately spaced 5-day courses of chloramphenicol therapy was not marked. In all instances a small increase in salivary colony counts occurred, but did not represent a major disturbance of the host-parasite equilibrium, and there was little change in the antibody level.

The results indicate that the model is closely analogous to the response to $C$. albicans observed in man. Further, it was possible to obtain detracted information about the dynamics of this response, and this information showed that where the response was stable chloramphenicol therapy did not precipitate invasive candidosis.

\section{LegionNAIRE'S Disease; SOME ENVIRONMENTAL ASPECTS}

\author{
J. B. Kurtz and M. Wills
}

Virology Laboratory, John Radcliffe Hospital, Oxford OX3 9DU

No environmental isolates of $L$. pneumophila were made, in spite of intensive investigation, in 
the original Philadelphia outbreak of Legionnaires' disease in July 1976. In 1978, however the organism was isolated from an air-conditioning cooling tower and from mud in a nearby thermally polluted stream, during the study of an outbreak in a students' complex at Bloomington, Indiana. This indicated that the organism existed in water. In 1979 piped water supplies were also implicated when $L$. pneumophilia was isolated from the filters and mixer valve of the shower in a room where two renal-transplant patients had become infected in Oxford.

This paper reviewed our experience of environmental isolates, and addressed itself to the problem of the symbiotic relationships of the organism.

\author{
33. DETECTION OF SPECIES-SPECIFIC AND CROSS-REACTIVE \\ CELL-SURFACE ANTIGENS OF BACTEROIDES BY AN ENZYME-LINKED \\ IMMUNOSORBENT ASSAY \\ I. R. Poxton, R. Brown and J. G. Collee \\ Department of Bacteriology, University of Edinburgh Medical School, \\ Edinburgh EH8 $9 A G$
}

Our basic lack of knowledge of the antigens of Bacteroides and the need for methods for the serological identification of Bacteroides to species level have prompted us to investigate their cell-surface antigens.

The EDTA-released outer-membrane antigen complexes were prepared from almost 200 laboratory and reference strains and 43 fresh clinical or faecal isolates representing 20 species or subspecies of Bacteroides, together with 12 species belonging to other genera. These antigens were titrated against antiserum to whole, live cells of 21 species or subspecies of Bacteroidaceae in an enzyme-linked immunosorbent assay (ELISA). The presence of species-specific antigens was investigated and cross reactions between species were noted. Results showed that a high proportion of the species possess species-specific antigens with little significant cross reactivity.

We believe that the ELISA technique described here detects antigens that are representative of the whole cell surface of bacteroides organisms. We have exploited the sensitivity of the technique and its quantitative potential to prepare guidelines for those wishing to use serological approaches for either the identification of Bacteroides species or for the titration of serum antibodies in a patient with a possible bacteroides infection.

\title{
34. ADHESION DETERMINANTS OF ESCHERICHIA COLI ISOLATED FROM URINARY-TRACT INFECTIONS
}

\author{
S. H. Parry, S. N. Abraham and M. Sussman \\ Department of Microbiology, University Medical School, \\ Newcastle upon Tyne NE1 7RU
}

Urinary-tract infections caused by $E$. coli involve colonisation of the bladder by organisms of faecal origin. A feature of this colonisation is the adherence of organisms to the epithelial surfaces. This is mediated by two types of fimbriae or pili, mannose-sensitive haemagglutinating or type-1 pili and mannose-resistant haemagglutinating (MRHA) pili. There is a strong correlation between the presence of MRHA pili and virulence in that $97 \%$ of $E$. coli isolated from patients with acute urinary-tract infections have MRHA pili while they are absent from E. coli isolated from the urethral flora of healthy women.

MRHA pili associated with urinary isolates can be distinguished from type-1 pili by their biological properties and their antigenic structure. Immunochemical analysis of MRHA-pilus antigens with specific antisera has been done and has indicated considerable antigenic heterogeneity with seven determinants $(a-g)$ occurring in combinations of $2-3$ on individual strains. Screening with determinant-specific antisera has demonstrated that the $c$ determinant is 
the most common ( $56 \%$ of isolates) and the $g$ determinant least common ( $12 \%$ of isolates). Detailed antigenic analysis of pilus antigens provides a new basis for investigation of the host response and may have a part to play in the serological identification of $E$. coli.

\title{
35. Studies ON CLOSTRIDIUM DIFFICILE
}

J. G. Collee, I. R. Poxton, Marie Byrne, R. Brown, G. Hay, R. Brettle and J. McC. Murdoch

Department of Bacteriology, University of Edinburgh, and Infectious Diseases Unit, City Hospital, Edinburgh

Methods for the rapid isolation and identification of Clostridium difficile from the faeces of patients with diarrhoea may be of wider clinical relevance than the recognition and management of classical pseudomembranous colitis.

The team's laboratory and hospital experience of $C$. difficile was reviewed, with special reference to the organism's selective and semi-quantitative isolation from faeces, its debated sensitivity to oxygen during processing on the bench, its saccharolytic profile, its toxigenicity, and difficulties experienced in the production of specific antitoxin. The organism's association with sporadic cases of diarrhoea in hospital parients was briefly reported, and its possible role in diarrhoeal disease was considered.

\section{WHAT IS THE REASON FOR THE PECULIAR COLONIES OF H. DUCREYI?}

\author{
M. G. McEntegart, S. Hafiz and G. Kinghorn* \\ Department of Medical Microbiology, Sheffield University Medical School \\ and Department of Genito-University Medicine,* Royal Infirmary, Sheffield
}

Ever since the first reports of the growth of $H$. ducreyi on solid medium, the peculiar coherent colonies have been a matter for comment. We have studied the structure of the colonies that can be pushed about the media without damage even to the edge of the colony. Several methods have been used ranging from conventional sections to scanning electron microscopy. We present our results and invite you to help us interpret. them.

\section{Viral GASTROENTERITIS IN VANCOUVER 1976-1980}

Donald M. McLean

\section{Medical Microbiology, University of British Columbia, Vancouver, Canada}

Electronmicroscopic examination of phosphotungstic-acid preparations of faecal extracts from 812 infants and childern aged less than 5 years who were admitted to the Children's Hospital, Vancouver during January 1976-December 1980 revealed virions in 213 patients (26.3\%). These included $16.8 \%$ rotaviruses, $5 \cdot 8 \%$ adenoviruses, $2 \cdot 2 \%$ astroviruses or caliciviruses and $1.5 \%$ picornavirus-like agents. Although virus excretion was usually brief, several patients revealed virus 5-7 days after onset. Gastroenteritis cases occurred more often during the winter months of December to March in each of the 5 years when virus excretion rates peaked at $31-40 \%$. Nosocomial spread of rotavirus gastroenteritis on open wards was halted promptly by rigorous observance of isolation procedures. 
68. YEAST-OPSONISING ACTIVITY IN NORMAL

AND PATHOLOGICAL SERA

\author{
A. Bashey, M. A. Kerr and J. Swanson Beck \\ Department of Pathology, Ninewells Hospital and Medical School, \\ Dundee DDI $9 S Y$
}

A simple quantitative assay of phagocytosis has been used to compare the ability of different sera to opsonise yeasts for uptake by isolated human polymorphonuclear neutrophils. The assay involves the incubation of killed yeast with neutrophils in 5\% serum and the estimation of free yeasts remaining in a Coulter counter and Channelyser.

In agreement with earlier studies, the sera of about $6 \%$ ( 2 from 34$)$ of healthy young people showed defective opsonisation. Investigation of the opsonising activity of 56 sera from healthy donors aged 65-88 selected at random from a Scottish urban general practice showed five deficient sera $(8.9 \%)$. The proportion of defective sera in the young and aged group was found to be not significantly different.

The opsonising activity of sera from 11 diseased young adults with low $\mathrm{C} 3$ levels was decreased. There was however no direct correlation between $\mathrm{C} 3$ levels and opsonising activity, and a single serum with low $\mathrm{C} 3(36 \mathrm{mg} \%$ ) showed apparently normal opsonising activity. 